\title{
Six New Induced Sesquiterpenes from the Cultures of Ascomycete Daldinia concentrica
}

\author{
Xiang-Dong Qin, Hong-Jun Shao, Ze-Jun Dong, Ji-Kai Liu
}

Received: April 23, 2008 / Accepted: August 19, 2008

(C) Japan Antibiotics Research Association

\begin{abstract}
Six new sesquiterpenes having the botryane carbon skeleton $(\mathbf{1} \sim \mathbf{6})$, together with known compounds (7 10) were induced and isolated from the ascomycete Daldinia concentrica (strain S 0318). Structures elucidation was accomplished by NMR spectroscopic and $\mathrm{X}$-ray crystallographic studies.
\end{abstract}

Keywords Sesquiterpenes, Daldinia concentrica, ascomycete, induction, botryane

\section{Introduction}

Fungi or bacteria that produce secondary metabolites often have the potential to produce various compounds from a single strain. Variation of cultivation parameters to induce the production of formerly unknown compounds is one of the approach to increase the number of secondary metabolites from one single organism. This way of releasing nature's chemical diversity was termed the 'OSMAC (One Strain-Many Compounds)' approach [1, 2], which is based on the observation that individual strains are able to produce more metabolites than normally detected in a routine screening program. Small changes in the cultivation parameters (for example, culture vessel, media composition, and addition of enzyme inhibitors) can completely shift the metabolic profile of various microorganisms. It is a very simple approach to the improvement of fermentations to obtain maximum

J.-K. Liu (Corresponding author), H.-J. Shao, X.-D. Qin, Z.-J. Dong: State Key Laboratory of Phytochemistry and Plant Resources in West China, Kunming Institute of Botany, Chinese Academy of Sciences, Kunming 650204, China, E-mail: jkliu@mail.kib.ac.cn production titers of desired compounds. This approach was successfully used as a valuable tool to exploit natural products diversity in the past, for example, actinomycetes (Streptomyces sp.) and fungi (Aspergillus sp., Sphaeropsidales sp.) produce additional compounds after variation of the culture conditions [3 5].

Previous investigation reported the isolation of induced daldinins $\mathrm{A}, \mathrm{B}$, and $\mathrm{C}$ with a new skeleton and four known compounds from the cultures of ascomycete Daldinia concentrica [6]. Following the OSMAC approach, the strain S 0318 (Daldinia concentrica) has been cultivated and further investigated. In the culture broth extract from Erlenmeyer flasks (color, transparent; size, $500 \mathrm{ml}$; media, $300 \mathrm{ml}$ ) instead of reagent bottles under same cultivation conditions we detected additional metabolites by TLC and HPLC. Careful investigation on the culture of $D$. concentrica strain (S 0318) led to the isolation of new compounds methyl-7 $\alpha$-acetoxydeacetylbotryoloate (1), $7 \alpha$ acetoxydeacetylbotryenedial (2), $7 \alpha$-hydroxybotryenalol

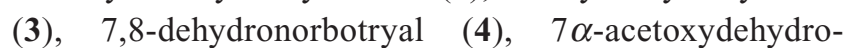
botrydienal (5), and $7 \alpha$-acetoxy-15-methoxy-10-O-methyldeacetyldihydrobotrydial (6) (Fig. 1), and known compounds, $7 \alpha$-hydroxy-10-O-methyldihydrobotrydial [7], 7-hydroxy-16- $O$-methyldeacetyldihydrobotrydial-hydrate [9, 10], 7-hydroxydeacetyl-botryenalol [8], and $7 \alpha$ hydroxydihydrobotrydial [7], by the alteration of culture parameter (form of culture vessel). The fermentation, isolation and structure elucidation of these compounds are reported here.

\section{Materials and Methods}

\section{General}

NMR experiments were performed on a Bruker AM-400 or 


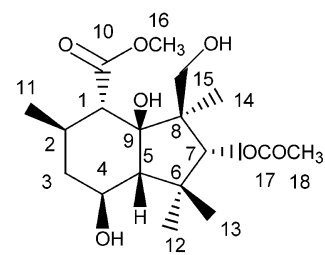

1

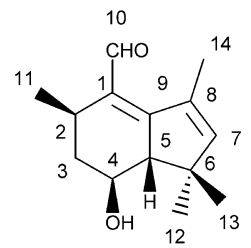

4

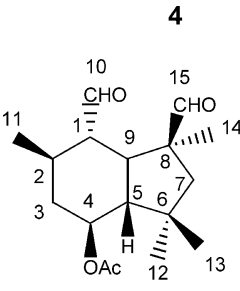

Botrydial

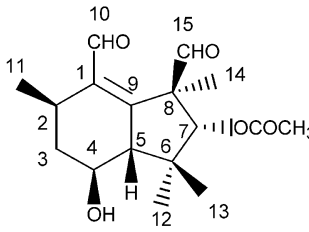

2

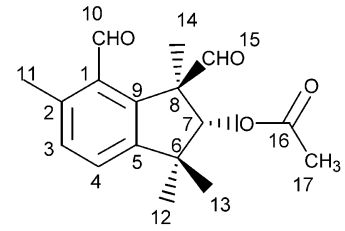

5

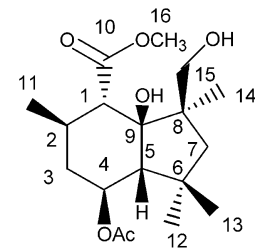

Methyl botryoloate

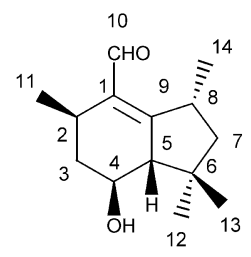

Norbotryal acetate
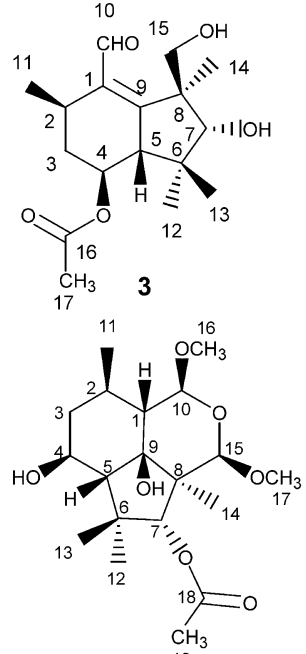

6

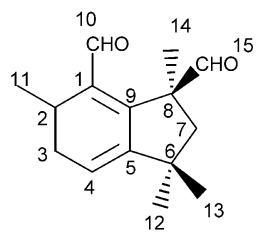

Botrydienal

Fig. 1 Structures of $\mathbf{1} \sim \mathbf{6}$.

1: methyl-7 $\alpha$-acetoxydeacetylbotryoloate, 2: $7 \alpha$-acetoxydeacetylbotryenedial, 3: $7 \alpha$-hydroxybotryenalol, 4: 7,8-dehydronorbotryal, 5 $7 \alpha$-acetoxydehydrobotrydienal, 6: $7 \alpha$-acetoxy-15-methoxy-10-O-methyl-deacetyldihydrobotrydial.

a DRX-500 spectrometer with TMS as internal standard. Mass spectra were recorded on a VG Auto Spec-3000 or an API QSTAR Pulsar 1 spectrometer. IR spectra were recorded on a Bruker Tensor 27 spectrometer with $\mathrm{KBr}$ pellets. Optical rotations were measured on a Horiba SEPA300 polarimeter. Column chromatography was performed on silica gel (200 300 mesh; Qingdao Marine Chemical Ltd., Qingdao, People's Republic of China) and Sephadex LH-20 (Amersham Biosciences, Sweden). TLC analysis was carried out on silica gel $\mathrm{GF}_{254}$ precoated plates ( $0.20 \sim 0.25 \mathrm{~mm}$; Qingdao) with detection by heating silica gel plates sprayed with $10 \% \mathrm{H}_{2} \mathrm{SO}_{4}$ in $\mathrm{EtOH}$.

\section{Fungal Material}

Strain (S 0318) was isolated from tissue culture of the fruiting bodies of Daldinia concentrica collected at Laojunshan, Yunnan Province, P. R. China, in July 2003 and identified by Prof. Mu Zang, Kunming Institute of Botany, Chinese Academy of Sciences. A voucher specimen (HKAS 40992) was deposited at the herbarium of Kunming Institute of Botany, Chinese Academy of Sciences.

\section{Cultivation}

Strain S 0318 was grown on agar slants at $22^{\circ} \mathrm{C}$ until sporulation, and then stored at $4^{\circ} \mathrm{C}$. The culture medium consisted of potato (peel off) $200 \mathrm{~g}$, glucose $20 \mathrm{~g}, \mathrm{KH}_{2} \mathrm{PO}_{4}$ $3.0 \mathrm{~g}, \mathrm{MgSO}_{4} 1.5 \mathrm{~g}$, citric acid $0.1 \mathrm{~g}$ and thiamin hydrochloride $10 \mathrm{mg}$ in 1.0 liter of de-ionized water. The fungus was grown in Erlenmeyer flasks (color, transparent; size, $500 \mathrm{ml}$; media, $300 \mathrm{ml}$ ). The $\mathrm{pH}$ was adjusted to 6.5 before autoclaving. Fermentation was carried out on a rotary shaker at $22^{\circ} \mathrm{C}$ and $150 \mathrm{rpm}$ for 10 days.

\section{Extraction and Isolation}

The mycelium from 60 liters was filtered, and the filtrate was extracted five times with EtOAc (total 80 liters). The EtOAc extract was evaporated in vacuo and the deep brown gum (80 g) was subjected to column chromatography (silica gel) using a $\mathrm{CHCl}_{3} / \mathrm{MeOH}$ stepwise elution. Elution with $\mathrm{CHCl}_{3} / \mathrm{MeOH}$ (98:2, v/v) produced 5 (11 mg). The fraction $(20 \mathrm{~g})$ from $\mathrm{CHCl}_{3} / \mathrm{MeOH}(95: 5)$ was further purified by repeated chromatography over silica gel (petroleum ether/ $\mathrm{Me}_{2} \mathrm{CO}$ from 20:1 to 10:1; $\mathrm{CHCl}_{3} / \mathrm{MeOH}$, from $30: 1$ to $10: 1$ ), and Sephadex LH-20 
$\left(\mathrm{CHCl}_{3} / \mathrm{MeOH}, 1: 1\right)$ to afford the pure $2(21 \mathrm{mg}), 3$ (35 mg), 4 (6 mg), 6 (27 mg), 8 (30 mg), $10(43 \mathrm{mg})$. The fraction (24 g) eluted with $\mathrm{CHCl}_{3} / \mathrm{MeOH}(90: 10)$ at the first chromatography was further purified by repeated chromatography over silica gel $\left(\mathrm{CHCl}_{3} / \mathrm{MeOH}\right.$, from $20: 1$ to $5: 1)$ to yield 1 (22 $\mathrm{mg}), 7$ (78 $\mathrm{mg}$ ), and $9(8 \mathrm{mg}$ ).

\section{Physico-chemical Properties}

Methyl-7 $\alpha$-acetoxydeacetylbotryoloate (1)

Colorless needles; m.p. $186^{\circ} \mathrm{C} ;[\alpha]_{\mathrm{D}}^{13}+2.52^{\circ}(c 0.53$ in $\mathrm{MeOH})$; IR (KBr) $v_{\max } 3353,2970,2957,1736,1710$, 1241, $1036 \mathrm{~cm}^{-1}$; UV (MeOH) $\lambda_{\max }(\log \varepsilon) 204(2.66) \mathrm{nm}$; EI-MS $m / z$ (rel intensity) $\left[\mathrm{M}-\mathrm{H}_{2} \mathrm{O}\right]^{+} 340$ (22), 309 (15), 280 (35), 265 (51), 250 (100), 235 (42), 210 (60), 185 (73), 153 (67), 109 (69), 95 (96), 83 (47); ${ }^{1} \mathrm{H}-$ and ${ }^{13} \mathrm{C}-\mathrm{NMR}$ data see Tables 1 and 3; Positive FAB-MS $m / z$ (rel intensity) $[\mathrm{M}+\mathrm{H}]^{+} 359$ (10); HR-ESI-MS $m / z[\mathrm{M}+\mathrm{Na}]^{+} 381.1885$ (calcd for $\mathrm{C}_{18} \mathrm{H}_{30} \mathrm{O}_{7} \mathrm{Na}, 381.1889$ ).

$7 \alpha$-Acetoxydeacetylbotryenedial (2)

Colorless crystal; m.p. $126^{\circ} \mathrm{C} ;[\alpha]_{\mathrm{D}}^{20}+211.9^{\circ}$ (c 0.23 in $\mathrm{MeOH}$ ); IR (KBr) $v_{\max } 3427,2980,2958,2933,2879$, $1714,1676,1264,1052 \mathrm{~cm}^{-1}$; UV $(\mathrm{MeOH}) \lambda_{\max }(\log \varepsilon)$ 252 (3.96) nm; ${ }^{1} \mathrm{H}$ - and ${ }^{13} \mathrm{C}-\mathrm{NMR}$ data see Tables 1 and 3; EI-MS $m / z$ (rel intensity) [M] 308 (3), 293 (7), 266 (2), 248 (20), 220 (65), 205 (65), 191 (80), 177 (55), 159 (100), 145 (30), 131 (27), 91 (32); Negative FAB-MS $m / z$ (rel intensity) $[\mathrm{M}-\mathrm{H}]^{-} 307$ (100); HR-TOF-MS $m / z$ : $[\mathrm{M}+\mathrm{H}]^{+}$
309.1706 (calcd for $\mathrm{C}_{17} \mathrm{H}_{24} \mathrm{O}_{5}, 309.1701$ ).

$7 \alpha$-Hydroxybotryenalol (3)

Colorless solid; m.p. $60^{\circ} \mathrm{C}$; $[\alpha]_{\mathrm{D}}^{21.6}+162.4^{\circ}$ (c 0.35 in $\mathrm{MeOH}$ ); IR (KBr) $v_{\max } 3472,2965,2936,2883,1713$, $1670,1267,1052,1042 \mathrm{~cm}^{-1}$; UV (MeOH) $\lambda_{\max }(\log \varepsilon)$ 252 (4.06) nm; ${ }^{1} \mathrm{H}$ - and ${ }^{13} \mathrm{C}-\mathrm{NMR}$ data see Tables 1 and 3; EI-MS $m / z$ (rel intensity) $[\mathrm{M}-\mathrm{H}]^{+} 309$ (3), 280 (12), 250 (5), 220 (44), 205 (55), 200 (100), 187 (35), 173 (40), 159 (38), 145 (18); Negative FAB-MS $m / z$ (rel intensity) $[\mathrm{M}-\mathrm{H}]^{-} 309$ (99); HR-TOF-MS $m / z$ : [M-H] 309.1700 (calcd for $\mathrm{C}_{17} \mathrm{H}_{25} \mathrm{O}_{5}, 309.1701$ ).

\section{7,8-Dehydronorbotryal (4)}

Colorless crystal; m.p. $112^{\circ} \mathrm{C} ;[\alpha]_{\mathrm{D}}^{24}-148.7^{\circ}(c 0.11$ in $\mathrm{MeOH}$ ); IR (KBr) $v_{\max } 3396,2957,2930,2867,1653$, 1594, $1063 \mathrm{~cm}^{-1}$; UV (MeOH) $\lambda_{\max }(\log \varepsilon) 294(4.31) \mathrm{nm}$; ${ }^{1} \mathrm{H}$ - and ${ }^{13} \mathrm{C}-\mathrm{NMR}$ data see Tables 2 and 3; EI-MS $m / z$ (rel intensity) $[\mathrm{M}+\mathrm{H}]^{+} 221$ (3), 185 (21), 167 (25), 149 (100), 69 (41); Positive TOF-MS $m / z$ (rel intensity) $[\mathrm{M}+\mathrm{H}]^{+} 221$; HR-TOF-MS $m / z[\mathrm{M}+\mathrm{H}]^{+} 221.1541$ (calcd for $\mathrm{C}_{14} \mathrm{H}_{20} \mathrm{O}_{2}$, 221.1541).

\section{$7 \alpha$-Acetoxydehydrobotrydienal (5)}

Colorless prisms; m.p. $162 \sim 163^{\circ} \mathrm{C} ;[\alpha]_{\mathrm{D}}^{26}-32.5^{\circ}(c 0.24$ in $\mathrm{MeOH}$ ); IR (KBr) $v_{\max } 2729,1728,1683,1591,1374$, $1254 \mathrm{~cm}^{-1}$; UV (MeOH) $\lambda_{\max }(\log \varepsilon) 204$ (4.33), 268 (3.27), 278 (2.16), 322 (2.58) nm; ${ }^{1} \mathrm{H}-$ and ${ }^{13} \mathrm{C}-\mathrm{NMR}$ data

Table $1{ }^{1}$ H-NMR data for $\mathbf{1} \sim \mathbf{3}$

\begin{tabular}{|c|c|c|c|}
\hline No. & 1 & 2 & 3 \\
\hline $\mathrm{H}-1$ & $2.38(1 \mathrm{H}, \mathrm{d}, J=12.3)$ & & \\
\hline $\mathrm{H}-2$ & $2.00(1 \mathrm{H}, \mathrm{m})$ & $2.82(1 \mathrm{H}, \mathrm{m}, J=3.8,6.7)$ & $2.87(1 \mathrm{H}, \mathrm{m})$ \\
\hline \multirow[t]{2}{*}{$\mathrm{H}-3$} & $\alpha 1.09(1 \mathrm{H}, \mathrm{m})$ & $\alpha 1.31(1 \mathrm{H}, \mathrm{m})$ & $\alpha 1.40(1 \mathrm{H}, \mathrm{ddd}, J=2.6,9.4,12.0)$ \\
\hline & $\beta 1.95(1 \mathrm{H}, \mathrm{m})$ & $\beta 2.11(1 \mathrm{H}, \mathrm{m})$ & $\beta 2.03(1 \mathrm{H}, \mathrm{ddd}, J=2.6,9.4,12.0)$ \\
\hline $\mathrm{H}-4$ & $3.88(1 \mathrm{H}, \mathrm{dt}, J=4.7,11.0)$ & $3.70(1 \mathrm{H}, \mathrm{ddd}, J=3.9,9.3,11.3)$ & $4.90(1 \mathrm{H}, \mathrm{m})$ \\
\hline$H-5$ & $1.59(1 \mathrm{H}, \mathrm{d}, J=11.0)$ & $2.42(1 \mathrm{H}, \mathrm{d}, J=9.3)$ & $2.44(1 \mathrm{H}, \mathrm{d}, \mathrm{J}=8.9)$ \\
\hline \multicolumn{4}{|l|}{$\mathrm{H}-6$} \\
\hline $\mathrm{H}-7$ & $5.48(1 \mathrm{H}, \mathrm{s})$ & $4.79(1 \mathrm{H}, \mathrm{s})$ & $3.67(1 \mathrm{H}, \mathrm{s})$ \\
\hline $\mathrm{H}-10$ & & $9.66(1 \mathrm{H}, \mathrm{s})$ & $10.24(1 \mathrm{H}, \mathrm{s})$ \\
\hline $\mathrm{H}-11$ & $0.91(3 \mathrm{H}, \mathrm{d}, J=6.1)$ & $1.02(3 \mathrm{H}, \mathrm{d}, J=6.7)$ & $1.07(3 \mathrm{H}, \mathrm{d}, J=6.8)$ \\
\hline $\mathrm{H}-12$ & $1.14(3 \mathrm{H}, \mathrm{s})$ & $1.01(3 \mathrm{H}, \mathrm{s})$ & $0.75(3 \mathrm{H}, \mathrm{s})$ \\
\hline $\mathrm{H}-13$ & $1.35(3 \mathrm{H}, \mathrm{s})$ & $1.30(3 \mathrm{H}, \mathrm{s})$ & $1.15(3 \mathrm{H}, \mathrm{s})$ \\
\hline $\mathrm{H}-14$ & $0.90(3 \mathrm{H}, \mathrm{s})$ & $1.28(3 \mathrm{H}, \mathrm{s})$ & $1.25(3 \mathrm{H}, \mathrm{s})$ \\
\hline \multirow[t]{2}{*}{$\mathrm{H}-15$} & $\alpha 3.19(1 \mathrm{H}, \mathrm{d}, J=11.9)$ & $9.52(1 \mathrm{H}, \mathrm{s})$ & $\alpha 3.56(1 \mathrm{H}, \mathrm{d}, J=10.7)$ \\
\hline & $\beta 3.44(1 \mathrm{H}, \mathrm{d}, J=11.9)$ & & $\beta 3.69(1 \mathrm{H}, \mathrm{d}, J=10.7)$ \\
\hline $\mathrm{H}-16$ & $3.73(3 \mathrm{H}, \mathrm{s})$ & & \\
\hline $\mathrm{H}-17$ & & $2.11(1 \mathrm{H}, \mathrm{s})$ & $2.05(3 \mathrm{H}, \mathrm{s})$ \\
\hline $\mathrm{H}-18$ & $2.09(3 \mathrm{H}, \mathrm{s})$ & & \\
\hline
\end{tabular}


Table $2{ }^{1}$ H-NMR data for $\mathbf{4} \sim \mathbf{6}$

\begin{tabular}{llll}
\hline No. & \multicolumn{1}{c}{$\mathbf{4}$} & \multicolumn{1}{c}{$\mathbf{5}$} & \multicolumn{1}{c}{$\mathbf{6}$} \\
\hline $\mathrm{H}-1$ & & & $1.46(1 \mathrm{H}, \mathrm{dd}, J=1.7,12.3)$ \\
$\mathrm{H}-2$ & $2.83(1 \mathrm{H}, \mathrm{m})$ & & $1.69(1 \mathrm{H}, \mathrm{m})$ \\
$\mathrm{H}-3$ & $\alpha 1.21(1 \mathrm{H}, \mathrm{ddd}, J=7.2,9.3,11.3)$ & $7.25(1 \mathrm{H}, \mathrm{d}, J=7.8)$ & $\alpha 1.10(1 \mathrm{H}, \mathrm{m})$ \\
& $\beta 2.13(1 \mathrm{H}, \mathrm{ddd}, J=3.9,7.2,11.3)$ & & $\beta 1.90(1 \mathrm{H}, \mathrm{m})$ \\
$\mathrm{H}-4$ & $3.63(1 \mathrm{H}, \mathrm{ddd}, J=3.9,10.0,11.3)$ & $7.33(1 \mathrm{H}, \mathrm{d}, J=7.8)$ & $3.96(1 \mathrm{H}, \mathrm{m})$ \\
$\mathrm{H}-5$ & $2.46(1 \mathrm{H}, \mathrm{d}, J=10.0)$ & & $1.58(1 \mathrm{H}, \mathrm{d}, J=10.0)$ \\
$\mathrm{H}-6$ & & & $5.35(1 \mathrm{H}, \mathrm{s})$ \\
$\mathrm{H}-7$ & $6.17(1 \mathrm{H}, \mathrm{s})$ & $4.95(1 \mathrm{H}, \mathrm{s})$ & $4.9(1 \mathrm{H}, \mathrm{d}, J=1.7)$ \\
$\mathrm{H}-10$ & $10.07(1 \mathrm{H}, \mathrm{s})$ & $10.36(1 \mathrm{H}, \mathrm{s})$ & $0.94(3 \mathrm{H}, \mathrm{d}, J=6.3)$ \\
$\mathrm{H}-11$ & $1.08(1 \mathrm{H}, \mathrm{d}, J=6.7)$ & $2.68(3 \mathrm{H}, \mathrm{s})$ & $1.15(3 \mathrm{H}, \mathrm{s})$ \\
$\mathrm{H}-12$ & $0.99(3 \mathrm{H}, \mathrm{s})$ & $1.24(3 \mathrm{H}, \mathrm{s})$ & $1.27(3 \mathrm{H}, \mathrm{s})$ \\
$\mathrm{H}-13$ & $1.22(3 \mathrm{H}, \mathrm{s})$ & $1.40(3 \mathrm{H}, \mathrm{s})$ & $0.97(3 \mathrm{H}, \mathrm{s})$ \\
$\mathrm{H}-14$ & $2.07(3 \mathrm{H}, \mathrm{s})$ & $1.44(3 \mathrm{H}, \mathrm{s})$ & $4.98(1 \mathrm{H}, \mathrm{s})$ \\
$\mathrm{H}-15$ & & $9.67(1 \mathrm{H}, \mathrm{s})$ & $3.44(3 \mathrm{H}, \mathrm{s})$ \\
$\mathrm{H}-16$ & & & $3.42(3 \mathrm{H}, \mathrm{s})$ \\
$\mathrm{H}-17$ & & $2.19(3 \mathrm{H}, \mathrm{s})$ & \\
$\mathrm{H}-18$ & & & $2.05(3 \mathrm{H}, \mathrm{s})$ \\
$\mathrm{H}-19$ & & &
\end{tabular}

Table $3{ }^{13} \mathrm{C}-\mathrm{NMR}$ data for $\mathbf{1} \sim \mathbf{6}$

\begin{tabular}{rrrrrrr}
\hline No. & $\mathbf{1}$ & $\mathbf{2}$ & $\mathbf{3}$ & $\mathbf{4}$ & $\mathbf{5}$ & \multicolumn{1}{c}{$\mathbf{6}$} \\
\hline 1 & 62.4 & 140.1 & 139.3 & 136.7 & 142.4 & 55.2 \\
2 & 30.8 & 31.5 & 30.3 & 31.2 & 128.7 & 29.1 \\
3 & 44.2 & 42.3 & 37.8 & 43.2 & 132.6 & 44.2 \\
3 A & & & & & & \\
4 & 70.0 & 68.3 & 71.5 & 68.9 & 128.7 & 69.5 \\
5 & 68.3 & 58.1 & 55.5 & 63.0 & 149.1 & 63.2 \\
6 & 40.3 & 43.5 & 43.6 & 46.4 & 44.6 & 41.1 \\
7 & 83.5 & 84.2 & 81.9 & 158.5 & 85.1 & 82.5 \\
8 & 52.2 & 59.0 & 50.7 & 136.7 & 59.5 & 51.0 \\
9 & 86.5 & 158.9 & 165.9 & 166.1 & 142.0 & 82.6 \\
10 & 175.4 & 193.7 & 194.8 & 191.7 & 190.7 & 100.6 \\
11 & 21.2 & 21.2 & 21.0 & 22.1 & 19.1 & 20.2 \\
12 & 21.0 & 17.6 & 16.7 & 24.0 & 26.2 & 20.9 \\
13 & 35.7 & 28.1 & 27.6 & 28.5 & 28.0 & 34.4 \\
14 & 14.8 & 18.7 & 22.1 & 19.2 & 15.3 & 12.0 \\
15 & 65.6 & 200.5 & 70.6 & & 201.8 & 102.0 \\
16 & 51.9 & 172.5 & 172.2 & & 170.8 & 55.4 \\
17 & 172.8 & 20.3 & 21.4 & & 20.6 & 56.8 \\
18 & 20.9 & & & & & 170.6 \\
19 & & & & & & 21.1 \\
\hline
\end{tabular}

see Tables 2 and 3; EI-MS $m / z$ (rel intensity) $[\mathrm{M}]^{+} 288$ (1), 217 (19), 200 (100), 185 (17), 172 (42), 157 (93); HR-ESIMS $m / z[\mathrm{M}+\mathrm{Na}]^{+} 311.1257$ (calcd for $\mathrm{C}_{17} \mathrm{H}_{20} \mathrm{O}_{4} \mathrm{Na}$,
311.1259).

$7 \alpha$-Acetoxy-15-methoxy-10-O-methyldeacetyldihydrobotrydial (6)

Colorless needles; m.p. $198 \sim 199^{\circ} \mathrm{C} ;[\alpha]_{\mathrm{D}}^{26}+85.2^{\circ}(c 0.9$ in $\mathrm{CHCl}_{3}$ ); IR (KBr) $v_{\max } 3461,3402,1742,1456,1370$, $1245 \mathrm{~cm}^{-1} ; \mathrm{UV}\left(\mathrm{CHCl}_{3}\right) \lambda_{\max }(\log \varepsilon) 219$ (2.6) nm; EI-MS $\mathrm{m} / z$ (rel intensity) $[\mathrm{M}]^{+} 371(1), 341(4), 220$ (15), 192 (40), 177 (30), 159 (22), 143 (80), 124 (33), 101 (100), 85 (61); Negative FAB-MS $m / z$ (rel intensity) $[\mathrm{M}-\mathrm{H}]^{-} 371$ (14); HR-TOF-MS $m / z[\mathrm{M}-\mathrm{H}]^{-} 371.2089$ (calcd for $\left.\mathrm{C}_{19} \mathrm{H}_{31} \mathrm{O}_{7}, 371.2069\right) ;{ }^{1} \mathrm{H}$ - and ${ }^{13} \mathrm{C}-\mathrm{NMR}$ data see Tables 2 and 3.

Crystallographic Data for $\mathbf{5}$

$\mathrm{C}_{17} \mathrm{H}_{20} \mathrm{O}_{4}, M 288.33$, orthohombic, space group $P 2{ }_{1} 2_{1} 2_{1}$, $a=10.613 \quad$ (2) $\AA, \quad b=11.153 \quad$ (2) $\AA, \quad c=13.143 \quad$ (3) $\AA$, $V=1555.7$ (5) $\AA^{3}, Z=4$, crystal dimensions $0.40 \times 0.40 \times$ $0.60 \mathrm{~mm}$ were used for measurements on a MAC DIP$2030 \mathrm{~K}$ diffractometer with a graphite monochromator $\left(\omega-2 \theta\right.$ scans, $\left.2 \theta_{\max }=50.0^{\circ}\right)$, Mo $\mathrm{K} \alpha$ radiation. The total number of independent reflections measured was 1640, of which 1559 were observed $\left(|F|^{2} \geq 2 \sigma|F|^{2}\right)$. Final indices: $R_{1}=0.0428, w R_{2}=0.1166$. The crystal structure (5) was solved by direct methods using SHELXS-97 and expanded using difference Fourier techniques, refined by the program and method $\mathrm{NOMCSDP}^{6}$ and full-matrix least-squares calculations. 
Crystallographic Data for 6

$\mathrm{C}_{19} \mathrm{H}_{32} \mathrm{O}_{7}, M 372.45$, orthohombic, space group $P 2{ }_{1}{ }_{2}{ }_{1}{ }_{1}$, $a=7.395 \quad$ (1) $\AA, \quad b=13.649 \quad$ (1) $\AA, \quad c=19.943 \quad$ (1) $\AA$, $V=2012.9$ (2) $\AA^{3}, Z=4$, crystal dimensions $0.15 \times 0.30 \times$ $0.40 \mathrm{~mm}$ were used for measurements on a MAC DIP$2030 \mathrm{~K}$ diffractometer with a graphite monochromator $\left(\omega-2 \theta\right.$ scans, $\left.2 \theta_{\max }=50.0^{\circ}\right)$, Mo $\mathrm{K} \alpha$ radiation. The total number of independent reflections measured was 2124, of which 1981 were observed $\left(|F|^{2} \geq 2 \sigma|F|^{2}\right)$. Final indices: $R_{1}=0.045, w R_{2}=0.130$. The crystal structure (6) was solved by direct methods using SHELXS-97 and expanded using difference Fourier techniques, refined by the program and method NOMCSDP ${ }^{6}$ and full-matrix least-squares calculations.

\section{Results and Discussion}

Compound 1 was obtained as colorless needles. The HRESI-MS of 1 gave an $[\mathrm{M}+\mathrm{Na}]^{+}$peak at $\mathrm{m} / z 381.1885$ (calcd. for $\mathrm{C}_{18} \mathrm{H}_{30} \mathrm{O}_{7} \mathrm{Na}$, 381.1889), which corresponded to a molecular formula of $\mathrm{C}_{18} \mathrm{H}_{30} \mathrm{O}_{7}$, requiring four degrees of unsaturation. IR spectral absorption at $3353 \mathrm{~cm}^{-1}$ indicated the presence of hydroxy group. The ${ }^{1} \mathrm{H}-\mathrm{NMR}$ spectrum exhibited a total of six methyl signals, i.e., five tertiary methyls at $\delta_{\mathrm{H}} 0.90$ (s, H-14), 1.14 (s, H-12), 1.35 (s, H-13), $2.09(\mathrm{~s}, \mathrm{H}-18)$, and $3.73(\mathrm{~s}, \mathrm{H}-16)$, and a methyl at $\delta_{\mathrm{H}} 0.91$ (d, $J=6.1 \mathrm{~Hz}, \mathrm{H}-11$ ). The ${ }^{13} \mathrm{C}-\mathrm{NMR}$ spectrum displayed 18 signals separated by DEPT experiments into six methyls (one for oxygenated methyl), two methylenes (one for oxygenated methylene), five methines (two for oxygenated methines), and five quaternary carbons (two for carbonyl carbons). Analysis of its HSQC and ${ }^{1} \mathrm{H}-{ }^{1} \mathrm{H}$ COSY led to the deduction of the fragment C-1-C-2-(C-11)-C-3-C-4-C-5. The NMR data suggested that $\mathbf{1}$ was similar in structure to the known compound methyl botryoloate, a phytotoxic botrydiane sesquiterpenoid isolated from Botrytis cinerea $[9,10]$. In fact, the main differences between the NMR spectral data of $\mathbf{1}$ and those of methyl botryoloate were that the methylene signal at $\delta_{\mathrm{C}} 52.2(\mathrm{t}, \mathrm{C}-7)$ in methyl botryoloate is replaced by an oxygenated methine signal at $\delta_{\mathrm{H}} 5.48(1 \mathrm{H}, \mathrm{s}, \mathrm{H}-7)$ and $\delta_{\mathrm{C}} 83.5(\mathrm{~d}, \mathrm{C}-7)$ in $\mathbf{1}$, and the chemical shift of H-4 in $\mathbf{1}$ was shifted upfield at $\delta_{\mathrm{H}} 5.10$ in methyl botryoloate to $\delta_{\mathrm{H}} 3.88$. The clearly correlation between $\mathrm{H}-7\left(\delta_{\mathrm{H}} 5.48\right)$ and $\mathrm{C}-17\left(\delta_{\mathrm{C}} 172.8\right)$ in the HMBC of 1 revealed that the acetoxy group take up position at C-7, and the stereochemistry of H-7 in $\mathbf{1}$ was established as $\alpha$ by means of ROESY experiments (Fig. 3). The assignments of 1 were further confirmed by analysis of combined ${ }^{1} \mathrm{H}-{ }^{1} \mathrm{H}$ COSY, HMBC and ROESY correlations (Fig. 3). Based on all those data described above, the structure of $\mathbf{1}$ was deduced to be methyl-7 $\alpha$-acetoxydeacetylbotryoloate as shown in Fig. 1.

The molecular formula, $\mathrm{C}_{17} \mathrm{H}_{24} \mathrm{O}_{5}$, for 2 was deduced from the HR-TOF-MS. The ${ }^{1} \mathrm{H}$ - and ${ }^{13} \mathrm{C}-\mathrm{NMR}$ spectra of $\mathbf{2}$ shared similarities with those of $\mathbf{1}$ and botryendial [9], except that the methylene at C-7 $\left(\delta_{\mathrm{H}} 1.52, \mathrm{~d}, J=13.2 \mathrm{~Hz}, \mathrm{H}-\right.$ $\left.7 \alpha ; \delta_{\mathrm{H}} 2.14, \mathrm{~d}, J=13.2 \mathrm{~Hz}, \mathrm{H}-7 \beta ; \delta_{\mathrm{C}} 51.1, \mathrm{t}, \mathrm{C}-7\right)$ in botryendial was replaced by an acetoxymethine $\left(\delta_{\mathrm{H}} 4.79\right.$, s, $\left.\mathrm{H}-7 ; \delta_{\mathrm{C}} 84.2, \mathrm{~d}, \mathrm{C}-7\right)$ in 2 . This was also supported by a cross peak observed between H-7 and C-16 $\left(\delta_{\mathrm{C}} 172.5\right)$ in the HMBC of 2. Meanwhile, in ${ }^{1} \mathrm{H}-\mathrm{NMR}$ spectrum, the methine signal of H-4 at $\delta_{\mathrm{H}} 4.93(\mathrm{ddd}, J=4.1,8.9,10.3 \mathrm{~Hz})$ in botryendial was observed upfield at $\delta_{\mathrm{H}} 3.70$ (ddd, $J=3.9$, $9.3,11.3 \mathrm{~Hz}$ ) in 2 . This suggested that the hydroxy group was located at C-4. Further, the structure of $\mathbf{2}$ was confirmed by combined ${ }^{1} \mathrm{H}-{ }^{1} \mathrm{H}$ COSY, HMBC and ROESY correlations analysis. Consequently, the structure of $\mathbf{2}$ was elucidated as $7 \alpha$-acetoxydeacetylbotryenedial.

Compound 3 possessed a molecular formula of $\mathrm{C}_{17} \mathrm{H}_{26} \mathrm{O}_{5}$ as determined by HR-TOF-MS and NMR data. The NMR spectra of $\mathbf{3}$ were nearly superimposable with those of $\mathbf{2}$, except for the emergence of an oxygenated methylene $\left(\delta_{\mathrm{H}}\right.$ $3.56, \mathrm{~d}, J=10.7 \mathrm{~Hz}, \mathrm{H}-15 \alpha ; \delta_{\mathrm{H}} 3.69, \mathrm{~d}, J=10.7 \mathrm{~Hz}, \mathrm{H}-15 \beta$; $\left.\delta_{\mathrm{C}} 70.6, \mathrm{t}, \mathrm{C}-15\right)$ and disappearance of the aldehyde group $\left(\delta_{\mathrm{H}} 9.52, \mathrm{~s}, \mathrm{H}-15 ; \delta_{\mathrm{C}} 200.5, \mathrm{~s}, \mathrm{C}-15\right)$. The acetoxy group was unambiguously attached to $\mathrm{C}-4$, which was concluded from the significant correlation between $\mathrm{H}-4\left(\delta_{\mathrm{H}} 4.90, \mathrm{~m}\right)$ and $\mathrm{C}-16\left(\delta_{\mathrm{C}} 172.2, \mathrm{~s}\right)$ in the HMBC. Analysis of the HSQC, ${ }^{1} \mathrm{H}^{-1} \mathrm{H}$ COSY, HMBC, and ROESY of 3 further enabled the establishment of its structure. Therefore, $\mathbf{3}$ was characterized as $7 \alpha$-hydroxybotryenalol.

Compound 4 was isolated as colorless crystals. The molecular formula was determined to be $\mathrm{C}_{14} \mathrm{H}_{20} \mathrm{O}_{2}$ by a combination of HR-TOF-MS and ${ }^{13} \mathrm{C}-\mathrm{NMR}$ data. Its IR spectrum revealed the presence of a hydroxyl $\left(3431 \mathrm{~cm}^{-1}\right)$ and an $\alpha, \beta$-unsaturated carbonyl $\left(2930,2867,1653 \mathrm{~cm}^{-1}\right)$ as functional groups. The ${ }^{1} \mathrm{H}-\mathrm{NMR}$ spectrum displayed signals attributable to four methyl groups $\left(\delta_{\mathrm{H}} 0.99, \mathrm{~s}, \mathrm{H}-12\right.$; $\delta_{\mathrm{H}} 1.08, \mathrm{~d}, J=6.7 \mathrm{~Hz}, \mathrm{H}-11 ; \delta_{\mathrm{H}} 1.22, \mathrm{~s}, \mathrm{H}-13 ; \delta_{\mathrm{H}} 2.07, \mathrm{~s}, \mathrm{H}-$ 14 ), a methylene ( $\delta_{\mathrm{H}} 1.21$, ddd, $J=7.2,9.3,11.3 \mathrm{~Hz}, \mathrm{H}-3 \alpha$; $\delta_{\mathrm{H}} 2.13$, ddd, $\left.J=3.9,7.2,11.3 \mathrm{~Hz}, \mathrm{H}-3 \beta\right)$, three methines $\left(\delta_{\mathrm{H}} 2.83, \mathrm{~m}, \mathrm{H}-2 ; \delta_{\mathrm{H}} 3.63\right.$, ddd, $J=3.9,9.3,11.3 \mathrm{~Hz}, \mathrm{H}-4$; $\left.\delta_{\mathrm{H}} 2.46, \mathrm{~d}, J=10.0 \mathrm{~Hz}, \mathrm{H}-5\right)$, a trisubstituted olefinic proton $\left(\delta_{\mathrm{H}} 6.17, \mathrm{~s}, \mathrm{H}-7\right)$, and a $\mathrm{CHO}$ group $\left(\delta_{\mathrm{H}} 10.07, \mathrm{~s}, \mathrm{H}-10\right)$. The 14 carbon signals were sorted into four methyls, a methylene and three $s p^{3}$ methines, one of which had an oxygen substitute at $\delta_{\mathrm{C}} 68.9(\mathrm{~d}, \mathrm{C}-4)$, an olefinic methine at $\delta_{\mathrm{C}} 158.5(\mathrm{~d}, \mathrm{C}-7)$ and an aldehyde methine at $\delta_{\mathrm{C}} 191.7(\mathrm{~d}$, $\mathrm{C}-10$ ), and four quaternary carbons, which contained three olefinic quaternary carbons at $\delta_{\mathrm{C}} 136.7$ (s, C-1), 136.7 (s, C-8), and 166.1 (s, C-9), respectively, by DEPT and HSQC 


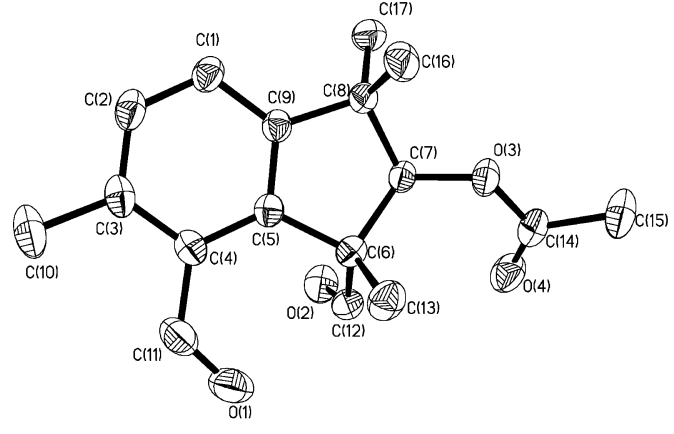

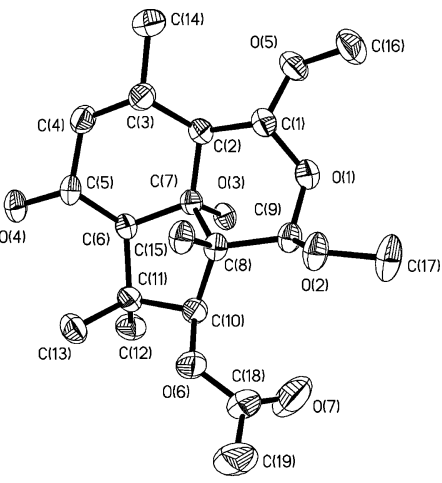

Fig. 2 Crystal structures of $\mathbf{5}$ and $\mathbf{6}$ with crystallographic numbering pattern.

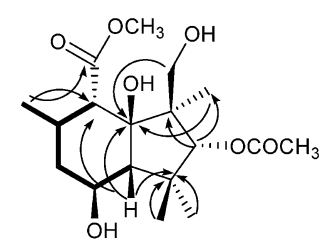

1

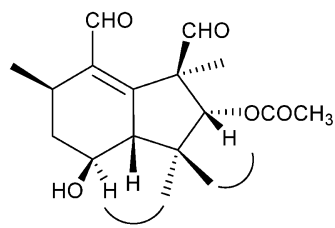

2

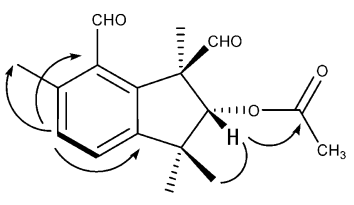

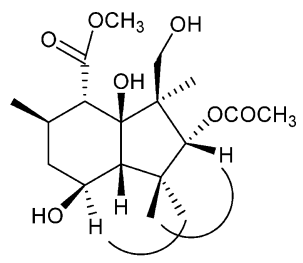

1
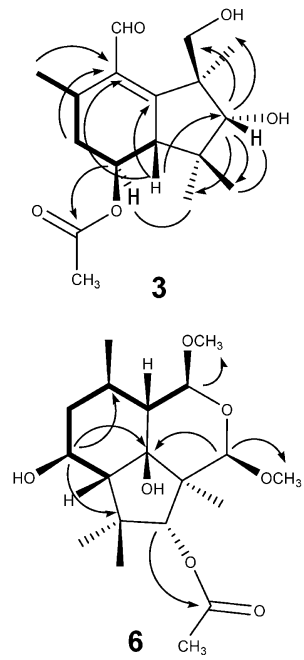

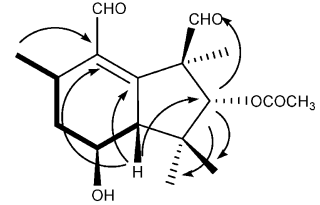

2

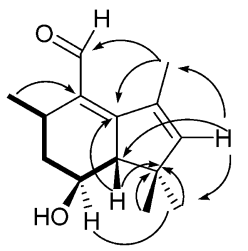

4

$-{ }^{1} \mathrm{H},{ }^{1} \mathrm{H}-\mathrm{COSY}$

$\longrightarrow \mathrm{HMBC}$

ROESY

Fig. 3 Key correlations of ${ }^{1} \mathrm{H},{ }^{1} \mathrm{H}-\mathrm{COSY}, \mathrm{HMBC}$ and ROESY for $\mathbf{1} \sim \mathbf{6}$.

experiments. Those above data suggested a bicyclic sesquiterpenoid skeleton for 4. Comparison of its NMR spectral data with those of norbotryal acetate, a norsesquiterpenoid aldehyde obtained from Botrytis cinerea, indicated that $\mathbf{4}$ also possessed the basic botryal skeleton, with the main difference being the appearance of a pair of double bond signal at $\delta_{\mathrm{H}} 6.17(\mathrm{~s}, \mathrm{H}-7), 158.5$ (d, C-7), and $\delta_{\mathrm{C}} 136.7$ (s, C-8) in the NMR spectrum, while the methylene signal at $\delta_{\mathrm{H}} 1.8(\mathrm{~m}, \mathrm{H}-7)$ and $\delta_{\mathrm{C}} 49.3(\mathrm{t}, \mathrm{C}-7)$, and the methine signal at $\delta_{\mathrm{H}} 3.2(\mathrm{~m}, \mathrm{H}-8)$ and $\delta_{\mathrm{C}} 29.1(\mathrm{t}, \mathrm{C}-$ 8) had disappeared. In addition, the chemical shift of $\mathrm{H}-4$ in 4 was shifted upfield from $\delta_{\mathrm{H}} 4.75$ in norbotryal acetate to $\delta_{\mathrm{H}}$ 3.63. This indicated that the hydroxy group was attached to $\mathrm{C}-4$. Further, the structure of $\mathbf{4}$ was substantiated by combined ${ }^{1} \mathrm{H}-{ }^{1} \mathrm{H}$ COSY, HMBC and ROESY correlations analysis (Fig. 3). Consequently, the structure of 4 was elucidated to be 7,8-dehydronorbotryal as shown. 
$7 \alpha$-Acetoxydehydrobotrydienal (5) was obtained as colorless prisms. The molecular formula of $\mathrm{C}_{17} \mathrm{H}_{20} \mathrm{O}_{4}$ was established on the basis of EI-MS, ${ }^{13} \mathrm{C}-\mathrm{NMR}$, DEPT NMR spectra and confirmed by HR-ESI-MS (found 311.1257, calcd for $\left.\mathrm{C}_{17} \mathrm{H}_{20} \mathrm{O}_{4} \mathrm{Na}, 311.1259\right)$. Its $\mathrm{UV}$ spectrum at $\lambda_{\text {max }}$ 204 (4.33), 268 (3.27), 278 (2.16), 322 (2.58) nm suggests that an aromatic ring is contained in $\mathbf{5}$. The ${ }^{13} \mathrm{C}-\mathrm{NMR}$ spectrum exhibited seventeen signals for five methyls ( $\delta$ 28.0, 26.2, 20.6, 19.1, and 15.3), five methines ( $\delta$ 201.8, $190.7,132.6,128.7$ and 85.1) and seven quarternary carbons $(\delta 170.8,149.1,142.4,142.0,128.7,59.5$ and 44.6). Its ${ }^{1} \mathrm{H}-\mathrm{NMR}$ spectrum (Table 2 ) was very close to that of dehydrobotrydienal [11], but the absence of doublet methane signals of $\mathrm{H}-7 \alpha$ and $\mathrm{H}-7 \beta$ and the appearance of two singlet signals at $\delta 4.95(1 \mathrm{H})$ and $2.19(3 \mathrm{H})$. The structure of $\mathbf{5}$ was suggested to be a 7-acetoxy compound of dehydrobotrydienal, which can be confirmed by HMBC correlations between H-7 $(\delta 4.95,1 \mathrm{H}, \mathrm{s}), 17(\delta 2.19,3 \mathrm{H}, \mathrm{s})$ and $\mathrm{C}-16$ ( $\delta$ 170.8). The orientation of $\mathrm{H}-7$ was inferred on the basis of NOESY. The correlations can be observed between $\mathrm{H}-7$ and $\mathrm{H}-13$, while no correlations were observed between H-7 and H-12, 14, suggesting that H-7 was $\beta$-orientated, which was confirmed with X-ray crystallographic analysis (Fig. 2).

$7 \alpha$-Acetoxy-15-methoxy-10-O-methyldeacetyldihydrobotrydial (6) was obtained as colorless needles. Its molecular formula of $\mathrm{C}_{19} \mathrm{H}_{32} \mathrm{O}_{7}$ was established on the basis of EI-MS, ${ }^{13} \mathrm{C}-\mathrm{NMR}$, and DEPT spectra and confirmed by HR-TOF-MS (found 371.2089, calcd for $\mathrm{C}_{19} \mathrm{H}_{31} \mathrm{O}_{7}$, 371.2069). The IR showed bands for acetate group (1742 and $\left.1245 \mathrm{~cm}^{-1}\right)$. The ${ }^{13} \mathrm{C}$-NMR spectrum exhibited nineteen signals for seven methyls ( $\delta 56.8,55.4,34.4,21.1$, $20.9,20.2$ and 12.0), one methylene ( $\delta$ 44.2), seven methines $(\delta 102.0,100.6,82.5,69.5,63.2,55.2$ and 29.1) and four quarternary carbons $(\delta 170.6,82.6,51.0$ and 41.1). Its NMR spectra (Table 2 ) were very close to that of 7-acetoxy-15-methoxy-10-O-methyldihydrobotrydial [10], but the signals for $\mathrm{H}-4$ and $\mathrm{C}-3,4,5$ with significant differences and the absence of an acetate group. In HMBC, correlations can be observed between $\mathrm{H}-4$ and $\mathrm{C}-5,6$, indicating one hydroxyl group connected to C-4. Thus structure of 6 was assigned to be 7-acetoxy-10-O-methyl15-methoxydeacetyldihydrobotrydial, which can be confirmed by X-ray crystallographic analysis (Fig. 2).

Natural products are an attractive source of varied structures that exhibit potent biological activities, and desirable pharmacological profiles. Variation of cultivation parameters to induce the production of formerly unknown compounds is one of the simple approach to increase the number of secondary metabolites from one single organism for fungi or bacteria. This way of releasing nature's chemical diversity could be further explored in the future.

Acknowledgments This work was supported by grants of CAS (KSCX1-YW-R-24 and KSCX2-YW-G-025) and National Basic Research Program of China (2009CB522301).

\section{References}

1. Bode HB, Bethe B, Hoefs R, Zeeck A. Big effects from small changes: possible ways to explore nature's chemical diversity. ChemBioChem 3: 619-627 (2002)

2. Hoefs R, Walker M, Zeeck A. Hexacyclinic acid, a polyketide from Streptomyces with a novel carbon skeleton. Angew Chem Int Ed 39: 3258-3261 (2000)

3. Fuchser J, Zeeck A. Secondary metabolites by chemical screening, 34. aspinolides and aspinonene/aspyrone cometabolites, new pentaketides produced by Aspergillus ochraceus. Liebigs Ann 87-95 (1997)

4. Grond S, Papastavrou I, Zeeck A. Studies of precursordirected biosynthesis with Streptomyces, 3 structural diversity of 1-O-acyl $\alpha$-L-rhamnopyranosides by precursordirected biosynthesis with Streptomyces griseoviridis. Eur J Org Chem 1875-1881 (2000)

5. Sattler I, Thiericke R, Zeeck A. The manumycin-group metabolites. Nat Prod Rep 15: 221-240 (1998)

6. Shao HJ, Qin XD, Dong ZJ, Zhang HB, Liu JK. Induced daldinin A, B, C with a new skeleton from cultures of the ascomycete Daldinia concentrica. J Antibiot 61: 115-119 (2008)

7. Thines E, Anke H, Steglich W, Sterner O. New botrydial sesquiterpenoids from Hymenoscyphus epiphyllus. Z Naturforsch Teil C 52: 413-416 (1997).

8. Krohn K, Dai JQ, Floerke U, Aust HJ, Draeger S, Schulz B. Botryane metabolites from the fungus Geniculosporium sp. isolated from the marine red alga Polysiphonia. J Nat Prod 68: 400-405 (2005)

9. Durán-Patrón R, Hernández-Galán R, Collado IG. Secobotrytriendiol and related sesquiterpenoids: new phytotoxic metabolites from Botrytis cinerea. J Nat Prod 63: 182-184 (2000)

10. Isidro GC, Rosario HG, Victoria P, James RH, Laureana GR. Biologically active sesquiterpenoid metabolites from the fungus Botrytis cinerea. Phytochemisty 41: 513-517 (1996)

11. Kimata T, Natsume M, Marumo S. Botrydienal, a new prytotoxin, and its related metabolites, dehydrobotrydienal and deacetyldihydrobotrydial produced by Botryotinia squamosa. Tetrahedron Lett 26: 2097-2100 (1985) 